\title{
Multichannel Deconvolution with Long-Range Dependence: A Minimax Study
}

\author{
Rida Benhaddou ${ }^{1}$, Rafal Kulik ${ }^{2}$, Marianna Pensky ${ }^{1}$ and Theofanis Sapatinas ${ }^{3}$ \\ ${ }^{1}$ Department of Mathematics, University of Central Florida, USA \\ ${ }^{2}$ Department of Mathematics and Statistics, University of Ottawa, Canada \\ ${ }^{3}$ Department of Mathematics and Statistics, University of Cyprus, Cyprus
}

\begin{abstract}
We consider the problem of estimating the unknown response function in the multichannel deconvolution model with long-range dependent Gaussian errors. We do not limit our consideration to a specific type of long-range dependence rather we assume that the errors should satisfy a general assumption in terms of the smallest and larger eigenvalues of their covariance matrices. We derive minimax lower bounds for the quadratic risk in the proposed multichannel deconvolution model when the response function is assumed to belong to a Besov ball and the blurring function is assumed to possess some smoothness properties, including both regular-smooth and super-smooth convolutions. Furthermore, we propose an adaptive wavelet estimator of the response function that is asymptotically optimal (in the minimax sense), or near-optimal within a logarithmic factor, in a wide range of Besov balls. It is shown that the optimal convergence rates depend on the balance between the smoothness parameter of the response function, the kernel parameters of the blurring function, the long memory parameters of the errors, and how the total number of observations is distributed among the total number of channels. Some examples of inverse problems in mathematical physics where one needs to recover initial or boundary conditions on the basis of observations from a noisy solution of a partial differential equation are used to illustrate the application of the theory we developed. The optimal convergence rates and the adaptive estimators we consider extend the ones studied by Pensky and Sapatinas $(2009,2010)$ for independent and identically distributed Gaussian errors to the case of long-range dependent Gaussian errors.
\end{abstract}

AMS (2000) Subject Classifications: 62G05 (primary), 62G08, 35J05, 35K05, 35L05 (secondary)

Keywords and Phrases: adaptivity, Besov spaces, block thresholding, deconvolution, Fourier analysis, functional data, long-range dependence, Meyer wavelets, minimax estimators, multichannel deconvolution, partial differential equations, stationary sequences, wavelet analysis. 


\section{Introduction}

We consider the estimation problem of the unknown response function $f(\cdot) \in L^{2}(T)$ from observations $y\left(u_{l}, t_{i}\right)$ driven by

$$
y\left(u_{l}, t_{i}\right)=\int_{T} g\left(u_{l}, t_{i}-x\right) f(x) d x+\xi_{l i}, \quad l=1,2, \ldots, M, i=1,2, \ldots, N,
$$

where $u_{l} \in U=[a, b], 0<a \leq b<\infty, T=[0,1], t_{i}=i / N$, and the errors $\xi_{l i}$ are Gaussian random variables, independent for different $l$ 's, but dependent for different $i$ 's.

Denote the total number of observations $n=N M$ and assume, without loss of generality, that $N=2^{J}$ for some integer $J>0$. For each $l=1,2, \ldots, M$, let $\boldsymbol{\xi}^{(l)}$ be a Gaussian vector with components $\xi_{l i}, i=1,2, \ldots, N$, and let $\boldsymbol{\Sigma}^{(l)}:=\operatorname{Cov}\left(\boldsymbol{\xi}^{(l)}\right):=\mathbb{E}\left[\boldsymbol{\xi}^{(l)}\left(\boldsymbol{\xi}^{(l)}\right)^{T}\right]$ be its covariance matrix.

Assumption A1: For each $l=1,2, \ldots, M, \boldsymbol{\Sigma}^{(l)}$ satisfies the following condition: there exist constants $K_{1}$ and $K_{2}\left(0<K_{1} \leq K_{2}<\infty\right)$, independent of $l$ and $N$, such that, for each $l=1,2, \ldots, M$,

$$
K_{1} N^{2 d_{l}} \leq \lambda_{\min }\left(\boldsymbol{\Sigma}^{(l)}\right) \leq \lambda_{\max }\left(\boldsymbol{\Sigma}^{(l)}\right) \leq K_{2} N^{2 d_{l}}, \quad 0 \leq d_{l}<1 / 2,
$$

where $\lambda_{\min }$ and $\lambda_{\max }$ are the smallest and the largest eigenvalues of (the Toeplitz matrix) $\boldsymbol{\Sigma}^{(l)}$. (Here, and in what follows, "T" denotes the transpose of a vector or a matrix.)

Assumption A1 is valid when, for each $l=1,2, \ldots, M, \boldsymbol{\xi}^{(l)}$ is a second-order stationary Gaussian sequence with spectral density satisfying certain assumptions. We shall elaborate on this issue in Section 2, Note that, in the case of independent errors, for each $l=1,2, \ldots, M, \boldsymbol{\Sigma}^{(l)}$ is proportional to the identity matrix and that $d_{l}=0$. In this case, the multichannel deconvolution model (1.1) reduces to the one with independent and identically distributed Gaussian errors. In a view of (1.1), the limit situation $d_{l}=0$, $l=1,2, \ldots, M$, can be thought of as the standard multichannel deconvolution model described in Pensky and Sapatinas (2009, 2010).

Model (1.1) can also be thought of as the discrete version of a model referred to as the functional deconvolution model by Pensky and Sapatinas $(2009,2010)$. The functional deconvolution model has a multitude of applications. In particular, it can be used in a number of inverse problems in mathematical physics where one needs to recover initial or boundary conditions on the basis of observations from a noisy solution of a partial differential equation. For instance, the problem of recovering the initial condition for parabolic equations based on observations in a fixed-time trip was first investigated in Lattes and Lions (1967), and the problem of recovering the boundary condition for elliptic equations based on observations in an interval domain was studied in Golubev and Khasminskii (1999) and Golubev (2004). 
In the case when $a=b$, the functional deconvolution model reduces to the standard deconvolution model. This model has been the subject of a great array of research papers since late 1980s, but the most significant contribution was that of Donoho (1995) who was the first to device a wavelet solution to the problem. This has attracted the attention of a good deal of researchers, see, e.g., Abramovich and Silverman (1998), Kalifa and Mallat (2003), Donoho and Raimondo (2004), Johnstone and Raimondo (2004), Johnstone, Kerkyacharian, Picard and Raimondo (2004), Kerkyacharian, Picard and Raimondo (2007). (For related results on the density deconvolution problem, we refer to, e.g., Pensky and Vidakovic (1999), Walter and Shen (1999), Fan and Koo (2002).)

In the multichannel deconvolution model studied by Pensky and Sapatinas (2009, 2010), as well as in the very current extension of their results to derivative estimation by Navarro et al. (2013), it is assumed that errors are independent and identically distributed Gaussian random variables. However, empirical evidence has shown that even at large lags, the correlation structure in the errors can decay at a hyperbolic rate, rather than an exponential rate. To account for this, a great deal of papers on long-range dependence (LRD) have been developed. The study of LRD (also called long memory) has a number of applications, as it can be reflected by the very large number of articles having LRD or long memory in their titles, in areas such as climate study, DNA sequencing, econometrics, finance, hydrology, internet modeling, signal and image processing, physics and even linguistics. Other applications can be found in Beran (1992, 1994), Beran et al. (2013) and Doukhan et al. (2003).

Although quite a few LRD models have been considered in the regression estimation framework, very little has been done in the standard deconvolution model. The density deconvolution set up has also witnessed some shift towards analyzing the problem for dependent processes. The argument behind that was that a number of statistical models, such as non-linear GARCH and continuous-time stochastic volatility models, can be looked at as density deconvolution models if we apply a simple logarithmic transformation, and thus there is need to account for dependence in the data. This started by Van Zanten et al. (2008) who investigated wavelet based density deconvolution studied by Pensky and Vidakovic (1999) with a relaxation to weakly dependent processes. Comte et al. (2008) analyzed another adaptive estimator that was proposed earlier but under the assumption that the sequence is strictly stationary but not necessarily independent. However, it was Kulik (2008), who considered the density deconvolution for LRD and short-range dependent (SRD) processes. However, Kulik (2008) did not considered nonlinear wavelet estimators but dealt instead with linear kernel estimators.

In nonparametric regression estimation, ARIMA-type models for the errors were analyzed in Cheng and Robinson (1994), with error terms of the form $\sigma\left(x_{i}, \xi_{i}\right)$. In Csörgo and Mielniczuk (2000), the error terms were modeled as infinite order moving averages processes. Mielniczuk and $\mathrm{Wu}$ (2004) investigated another form of LRD, with the assumption that $x_{i}$ and $\xi_{i}$ are not necessarily independent for the same $i$. ARIMA-type error models were also considered in Kulik and Raimondo (2009). In the standard deconvolution model, 
and using a maxiset approach, Wishart (2012) applied a fractional Brownian motion to model the presence of LRD, while Wang (2012) used a minimax approach to study the problem of recovering a function $f$ from a more general noisy linear transformation where the noise is also a fractional Brownian motion.

The objective of this paper is to study the multichannel deconvolution model from a minimax point of view, with the relaxation that errors exhibit LRD. We do not limit our consideration to a specific type of LRD: the only restriction is that the errors should satisfy Assumption A1. In particular, we derive minimax lower bounds for the $L^{2}$-risk in model (1.1) under Assumption A1 when $f(\cdot)$ is assumed to belong to a Besov ball and $g(\cdot, \cdot)$ has smoothness properties similar to those in Pensky and Sapatinas (2009, 2010), including both regular-smooth and super-smooth convolutions. In addition, we propose an adaptive wavelet estimator for $f(\cdot)$ and show that such estimator is asymptotically optimal (or near-optimal within a logarithmic factor) in the minimax sense, in a wide range of Besov balls. We prove that the convergence rates of the resulting estimators depend on the balance between the smoothness parameter (of the response function $f(\cdot)$ ), the kernel parameters (of the blurring function $g(\cdot, \cdot)$ ), and the long memory parameters $d_{l}$, $l=1,2 \ldots, M$ (of the error sequence $\boldsymbol{\xi}^{(l)}$ ). Since the parameters $d_{l}$ depend on the values of $l$, the convergence rates have more complex expressions than the ones obtained in Kulik and Raimondo (2009) when studying nonparametric regression estimation with ARIMAtype error models. The convergence rates we derive are more similar in nature to those in Pensky and Sapatinas $(2009,2010)$. In particular, the convergence rates depend on how the total number $n=N M$ of observations is distributed among the total number $M$ of channels. As we illustrate in two examples, convergence rates are not affected by long range dependence in case of super-smooth convolutions, however, the situation changes in regular cases.

The paper is organized as follows. Section 2 discusses stationary sequences with LRD errors, justifies Assumption A1 and provides illustrative examples of stationary sequences satisfying this assumption. Section 3 describes the construction of the suggested wavelet estimator of $f(\cdot)$. Section 4 derives minimax lower bounds for the $L^{2}$-risk for observations from model (1.1). Section 5 proves that the suggested wavelet estimator is adaptive and asymptotically optimal (in the minimax sense) or near-optimal within a logarithmic factor, in a wide range of Besov balls. Section 6 presents examples of inverse problems in mathematical physics where one needs to recover initial or boundary conditions on the basis of observations from a noisy solution of a partial differential equation to illustrate the application of the theory we developed. Section 7 concludes with a brief discussion. Section 8 contains the proofs of the theoretical results obtained in earlier sections.

\section{Stationary Sequences with Long-Range Dependence}

In this section, for simplicity of exposition, we consider one sequence of errors $\left\{\xi_{j}: j=\right.$ $1,2, \ldots\}$. Assume that $\left\{\xi_{j}: j=1,2, \ldots\right\}$ is a second-order stationary sequence with 
covariance function $\gamma_{\xi}(k):=\gamma(k), k=0, \pm 1, \pm 2, \ldots$. The spectral density is defined as

$$
a_{\xi}(\lambda):=a(\lambda):=\frac{1}{2 \pi} \sum_{k=-\infty}^{\infty} \gamma(k) \exp (-i k \lambda), \quad \lambda \in[-\pi, \pi] .
$$

On the other hand, the inverse transform which recovers $\gamma(k), k=0, \pm 1, \pm 2, \ldots$, from $a(\lambda), \lambda \in[-\pi, \pi]$, is given by

$$
\gamma(k)=\int_{-\pi}^{\pi} e^{i k \lambda} a(\lambda) d \lambda, \quad k=0, \pm 1, \pm 2, \ldots,
$$

under the assumption that the spectral density $a(\lambda), \lambda \in[-\pi, \pi]$, is squared-integrable.

Let $\boldsymbol{\Sigma}=[\gamma(j-k)]_{j, k=1}^{N}$ be the covariance matrix of $\left(\xi_{1}, \ldots, \xi_{N}\right)$. Define $\mathcal{X}=\{\mathbf{x} \in$ $\left.\mathbb{C}^{N}: \mathbf{x}^{*} \mathbf{x}=1\right\}$, where $\mathbf{x}^{*}$ is the complex-conjugate of $\mathbf{x}$. Since $\boldsymbol{\Sigma}$ is Hermitian, one has

$$
\lambda_{\min }(\boldsymbol{\Sigma})=\inf _{\mathbf{x} \in \mathcal{X}}\left(\mathbf{x}^{*} \boldsymbol{\Sigma} \mathbf{x}\right) \quad \text { and } \quad \lambda_{\max }(\boldsymbol{\Sigma})=\sup _{\mathbf{x} \in \mathcal{X}}\left(\mathbf{x}^{*} \boldsymbol{\Sigma} \mathbf{x}\right) .
$$

With the definitions introduced above,

$$
\mathbf{x}^{*} \mathbf{\Sigma} \mathbf{x}=\sum_{j, k=1}^{N} \mathbf{x}^{*} \gamma(j-k) \mathbf{x}=\int_{-\pi}^{\pi}\left|\sum_{j=1}^{N} x_{j} e^{-i j \lambda}\right|^{2} a(\lambda) d \lambda .
$$

Note that, by the Parseval identity, the function $h(\lambda)=\left|\sum_{j=1}^{N} x_{j} e^{-i j \lambda}\right|^{2}, \lambda \in[-\pi, \pi]$, belongs to the set

$$
\mathcal{H}_{N}=\left\{h: h \text { symmetric, }|h|_{\infty} \leq N, \int_{-\pi}^{\pi} h(\lambda) d \lambda=2 \pi\right\} .
$$

Let $d \in[0,1 / 2)$. Consider the following class of spectral densities

$$
\mathcal{F}_{d}=\left\{a: a(\lambda)=|\lambda|^{-2 d} a_{*}(\lambda), 0<C_{\min } \leq\left|a_{*}(\lambda)\right| \leq C_{\max }<\infty, \lambda \in[-\pi, \pi]\right\} .
$$

Below we provide two examples of second-order stationary sequences such that their spectral densities $a(\lambda), \lambda \in[-\pi, \pi]$, belong to the class $\mathcal{F}_{d}$ described in (2.3) .

Fractional $\operatorname{ARIMA}(\mathbf{0}, d, \mathbf{0})$. Let $\left\{\xi_{j}: j=1,2, \ldots\right\}$ be the second-order stationary sequence

$$
\xi_{j}=\sum_{m=0}^{\infty} a_{m} \eta_{j-m}
$$

where $\eta_{j}$ are uncorrelated, zero-mean, random variables, $\sigma_{\eta}^{2}:=\operatorname{Var}\left(\eta_{j}\right)<\infty$, and

$$
a_{m}=(-1)^{m}\left(\begin{array}{c}
-d \\
m
\end{array}\right)=(-1)^{m} \frac{\Gamma(1-d)}{\Gamma(m+1) \Gamma(1-d-m)}
$$


with $d \in[0,1 / 2)$. Then, $a_{m}, m=0,1, \ldots$, are the coefficients in the power-series representation

$$
A(z):=(1-z)^{-d}:=\sum_{m=0}^{\infty} a_{m} z^{m}
$$

Therefore, the spectral density $a(\lambda), \lambda \in[-\pi, \pi]$, of $\left\{\xi_{j}: j=1,2, \ldots\right\}$, is given by

$$
a(\lambda)=\frac{\sigma_{\eta}^{2}}{2 \pi}\left|A\left(e^{-i \lambda}\right)\right|^{2}=\frac{\sigma_{\eta}^{2}}{2 \pi}\left|1-e^{-i \lambda}\right|^{-2 d}=\frac{\sigma_{\eta}^{2}}{2 \pi}|2(1-\cos \lambda)|^{-d} \sim \frac{\sigma_{\eta}^{2}}{2 \pi}|\lambda|^{-2 d}(\lambda \rightarrow 0) .
$$

Hence, the sequence $\left\{\xi_{j}: j=1,2, \ldots\right\}$ has spectral density $a(\lambda), \lambda \in[-\pi, \pi]$, that belongs to the class $\mathcal{F}_{d}$ described in (2.3). (The sequence $\left\{\xi_{j}: j=1,2, \ldots\right\}$ is called the fractional $\operatorname{ARIMA}(0, d, 0)$ time series.)

Fractional Gaussian Noise. Assume that $B_{H}(u), u \in[0, \infty]$, is a fractional Brownian motion with the Hurst parameter $H \in[1 / 2,1)$. Define the second-order stationary sequence $\xi_{j}=B_{H}(j)-B_{H}(j-1), j=1,2, \ldots$. Its spectral density $a(\lambda), \lambda \in[-\pi, \pi]$, is given by (see, e.g., [14], p. 222)

$$
a(\lambda)=\sigma^{2}(2 \pi)^{-2 H-2} \Gamma(2 H+1) \sin (\pi H) 4 \sin ^{2}(\lambda / 2) \times \sum_{k=-\infty}^{\infty}|k+(\lambda / 2 \pi)|^{-2 H-1},
$$

and, hence,

$$
a(\lambda)=\frac{2 \sigma^{2}}{\pi} \Gamma(2 H+1) \sin (\pi H) \lambda^{1-2 H} \quad(\lambda \rightarrow 0) .
$$

Hence, the sequence $\left\{\xi_{j}: j=1,2, \ldots\right\}$ has spectral density $a(\lambda), \lambda \in[-\pi, \pi]$, that belongs to class $\mathcal{F}_{d}$ with $d=H-1 / 2$. (The sequence $\left\{\xi_{j}: j=1,2, \ldots\right\}$ is called the fractional Gaussian noise.)

It follows from (2.3) that, for $a \in \mathcal{F}_{d}$, one has $a(\lambda) \sim|\lambda|^{-2 d}(\lambda \rightarrow 0)$. It also turns out that the condition $a \in \mathcal{F}_{d}, d \in[0,1 / 2)$, implies that all eigenvalues of the covariance matrix $\boldsymbol{\Sigma}$ are of asymptotic order $N^{2 d}(N \rightarrow \infty)$. In particular, the following lemma is true.

Lemma 1 Assume that $\left\{\xi_{j}: j=1,2, \ldots\right\}$ is a second-order stationary sequence with spectral density $a \in \mathcal{F}_{d}, d \in[0,1 / 2)$. Then, for some constants $K_{1 d}$ and $K_{2 d}\left(0<K_{1 d} \leq\right.$ $\left.K_{2 d}<\infty\right)$, that depend on $d$ only,

$$
K_{1 d} N^{2 d} \leq \lambda_{\min }(\boldsymbol{\Sigma}) \leq \lambda_{\max }(\boldsymbol{\Sigma}) \leq K_{2 d} N^{2 d}
$$

Remark 1 If $d=0$, then $\mathcal{F}_{d}$ is the class of spectral densities $a(\lambda)$ that are bounded away from 0 and $\infty$ for all $\lambda \in[-\pi, \pi]$. In particular, the corresponding second-order stationary sequences $\left\{\xi_{j}: j=1,2, \ldots\right\}$ are weakly dependent. Then, the statement of Lemma 1 reduces to a result in Grenander and Szegö [17], Section 5.2. 
It follows immediately from Lemma 1 that if, for each $l=1,2, \ldots, M, \boldsymbol{\xi}^{(l)}$ is a secondorder stationary Gaussian sequence with spectral density $a_{l} \in \mathcal{F}_{d_{l}}, d_{l} \in[0,1 / 2)$, that $\boldsymbol{\xi}^{(l)}$ are independent for different $l$ 's, and that $d_{l}$ 's are uniformly bounded, then Assumption A1 holds.

Corollary 1 For each $l=1,2, \ldots, M$, let $\boldsymbol{\xi}^{(l)}$ be a second-order stationary Gaussian sequence with spectral density $a_{l} \in \mathcal{F}_{d_{l}}, d_{l} \in[0,1 / 2)$. We assume that $\boldsymbol{\xi}^{(l)}$ are independent for different l's. Let $d_{l}, l=1,2, \ldots, M$, be uniformly bounded, i.e., there exists $d^{*}(0 \leq$ $\left.d^{*}<1 / 2\right)$ such that, for each $l=1,2, \ldots, M$,

$$
0 \leq d_{l} \leq d^{*}<1 / 2
$$

Then, Assumption A1 holds.

\section{The Estimation Algorithm}

In what follows, $\langle\cdot, \cdot\rangle$ denotes the inner product in $\mathbb{R}^{N}$. We also denote the complexconjugate of $a \in \mathbb{C}$ by $\bar{a}$, the discrete Fourier basis on the interval $T$ by $e_{m}\left(t_{i}\right)=e^{-i 2 \pi m t_{i}}$, $t_{i}=i / N, i=1,2, \ldots, N, m=0, \pm 1, \pm 2, \ldots$, and the complex-conjugate of the matrix $\mathbf{A}$ by $\mathbf{A}^{*}$.

Recall the multichannel deconvolution model (1.1). Denote

$$
h\left(u_{l}, t_{i}\right)=\int_{T} g\left(u_{l}, t_{i}-x\right) f(x) d x, \quad l=1,2, \ldots, M, \quad i=1,2, \ldots, N .
$$

Then, equation (1.1) can be rewritten as

$$
y\left(u_{l}, t_{i}\right)=h\left(u_{l}, t_{i}\right)+\xi_{l i}, \quad l=1,2, \ldots, M, \quad i=1,2, \ldots, N .
$$

For each $l=1,2, \ldots, M$, let $h_{m}\left(u_{l}\right)=\left\langle e_{m}, h\left(u_{l}, \cdot\right)\right\rangle, y_{m}\left(u_{l}\right)=\left\langle e_{m}, y\left(u_{l}, \cdot\right)\right\rangle, z_{l m}=$ $\left\langle e_{m}, \boldsymbol{\xi}^{(l)}\right\rangle, g_{m}\left(u_{l}\right)=\left\langle e_{m}, g\left(u_{l}, \cdot\right)\right\rangle$ and $f_{m}=\left\langle e_{m}, f\right\rangle$ be the discrete Fourier coefficients of the $\mathbb{R}^{N}$ vectors $h\left(u_{l}, t_{i}\right), y\left(u_{l}, t_{i}\right), \xi_{l i}, g\left(u_{l}, t_{i}\right)$ and $f\left(t_{i}\right), i=1,2, \ldots, N$, respectively. Then, applying the discrete Fourier transform to (3.1), one obtains, for any $u_{l} \in U$, $l=1,2, \ldots, M$,

$$
y_{m}\left(u_{l}\right)=g_{m}\left(u_{l}\right) f_{m}+N^{-1 / 2} z_{l m}
$$

and

$$
h_{m}\left(u_{l}\right)=g_{m}\left(u_{l}\right) f_{m} .
$$

Multiplying both sides of (3.2) by $N^{-2} d_{l} \overline{g_{m}\left(u_{l}\right)}$, and adding them together, we obtain the following estimator of $f_{m}$

$$
\widehat{f}_{m}=\left(\sum_{l=1}^{M} N^{-2 d_{l}} \overline{g_{m}\left(u_{l}\right)} y_{m}\left(u_{l}\right)\right) /\left(\sum_{l=1}^{M} N^{-2 d_{l}}\left|g_{m}\left(u_{l}\right)\right|^{2}\right) .
$$


Let $\varphi^{*}(\cdot)$ and $\psi^{*}(\cdot)$ be the Meyer scaling and mother wavelet functions, respectively, defined on the real line (see, e.g., Meyer (1992)), and obtain a periodized version of Meyer wavelet basis as in Johnstone et al. (2004), i.e., for $j \geq 0$ and $k=0,1, \ldots, 2^{j}-1$,

$$
\varphi_{j k}(x)=\sum_{i \in \mathbb{Z}} 2^{j / 2} \varphi^{*}\left(2^{j}(x+i)-k\right), \quad \psi_{j k}(x)=\sum_{i \in \mathbb{Z}} 2^{j / 2} \psi^{*}\left(2^{j}(x+i)-k\right), \quad x \in T .
$$

Following Pensky and Sapatinas (2009, 2010), using the periodized Meyer wavelet basis described above, for some $j_{0} \geq 0$, expand $f(\cdot) \in L^{2}(T)$ as

$$
f(t)=\sum_{k=0}^{2^{j_{0}}-1} a_{j_{0} k} \varphi_{j_{0} k}(t)+\sum_{j=j_{0}}^{\infty} \sum_{k=0}^{2^{j}-1} b_{j k} \psi_{j k}(t), \quad t \in T .
$$

Furthermore, by Plancherel's formula, the scaling coefficients, $a_{j_{0} k}=\left\langle f, \varphi_{j_{0} k}\right\rangle$, and the wavelet coefficients, $b_{j k}=\left\langle f, \psi_{j k}\right\rangle$, of $f(\cdot)$ can be represented as

$$
a_{j_{0} k}=\sum_{m \in C_{j_{0}}} f_{m} \overline{\varphi_{m j_{0} k}}, \quad b_{j k}=\sum_{m \in C_{j}} f_{m} \overline{\psi_{m j k}}
$$

where $C_{j_{0}}=\left\{m: \varphi_{m j_{0} k} \neq 0\right\}$ and, for any $j \geq j_{0}$,

$$
C_{j}=\left\{m: \psi_{m j k} \neq 0\right\} \subseteq 2 \pi / 3\left[-2^{j+2},-2^{j}\right] \cup\left[2^{j}, 2^{j+2}\right] .
$$

(Note that the cardinality $\left|C_{j}\right|$ of the set $C_{j}$ is $\left|C_{j}\right|=4 \pi 2^{j}$, see, e.g., Johnstone et al. (2004).) Estimates of $a_{j_{0} k}$ and $b_{j k}$ are readily obtained by substituting $f_{m}$ in (3.6) with (3.4), i.e.,

$$
\widehat{a}_{j_{0} k}=\sum_{m \in C_{j_{0}}} \widehat{f}_{m} \overline{\varphi_{m j_{0} k}}, \quad \widehat{b}_{j k}=\sum_{m \in C_{j}} \widehat{f}_{m} \overline{\psi_{m j k}} .
$$

We now construct a (block thresholding) wavelet estimator of $f(\cdot)$, suggested by Pensky \& Sapatinas $(2009,2010)$. For this purpose, we divide the wavelet coefficients at each resolution level into blocks of length $\ln n$. Let $A_{j}$ and $U_{j r}$ be the following sets of indices

$$
\begin{gathered}
A_{j}=\left\{r \mid r=1,2, \ldots, 2^{j} / \ln n\right\}, \\
U_{j r}=\left\{k \mid k=0,1, \ldots, 2^{j}-1 ;(r-1) \ln n \leq k \leq r \ln n-1\right\} .
\end{gathered}
$$

Denote

$$
B_{j r}=\sum_{k \in U_{j r}} b_{j k}^{2}, \quad \widehat{B}_{j r}=\sum_{k \in U_{j r}} \widehat{b}_{j k}^{2} .
$$

Finally, for any $j_{0} \geq 0$, the (block thresholding) wavelet estimator $\hat{f}_{n}(\cdot)$ of $f(\cdot)$ is constructed as

$$
\hat{f}_{n}(t)=\sum_{k=0}^{2^{j_{0}}-1} \widehat{a}_{j_{0} k} \varphi_{j_{0} k}(t)+\sum_{j=j_{0}}^{J-1} \sum_{r \in A_{j}} \sum_{k \in U_{j r}} \widehat{b}_{j k} \mathbb{I}\left(\left|\widehat{B}_{j r}\right| \geq \lambda_{j}\right) \psi_{j k}(t), \quad t \in T,
$$


where $\mathbb{I}(A)$ is the indicator function of the set $A$, and the resolution levels $j_{0}$ and $J$ and the thresholds $\lambda_{j}$ will be defined in Section 5 .

In what follows, the symbol $C$ is used for a generic positive constant, independent of $n$, while the symbol $K$ is used for a generic positive constant, independent of $m, n, M$ and $u_{1}, u_{2}, \ldots, u_{M}$. Either of $C$ or $K$ may take different values at different places.

\section{Minimax Lower Bounds for the $L^{2}$-Risk}

Denote

$$
s^{\prime}=s+1 / 2-1 / p, \quad s^{*}=s+1 / 2-1 / p^{\prime}, \quad p^{\prime}=\min \{p, 2\} .
$$

Assume that the unknown response function $f(\cdot)$ belongs to a Besov ball $B_{p, q}^{s}(A)$ of radius $A>0$, so that the wavelet coefficients $a_{j_{0} k}$ and $b_{j k}$ defined in (3.6) satisfy the following relation

$$
B_{p, q}^{s}(A)=\left\{f \in L^{2}(U):\left(\sum_{k=0}^{2^{j} 0-1}\left|a_{j_{0} k}\right|^{p}\right)^{\frac{1}{p}}+\left(\sum_{j=j_{0}}^{\infty} 2^{j s^{\prime} q}\left(\sum_{k=0}^{2^{j}-1}\left|b_{j k}\right|^{p}\right)^{\frac{q}{p}}\right)^{1 / q} \leq A\right\} .
$$

Below, we construct minimax lower bounds for the (quadratic) $L^{2}$-risk. For this purpose, we define the minimax $L^{2}$-risk over the set $V \subseteq L^{2}(T)$ as

$$
R_{n}(V)=\inf _{\tilde{f}} \sup _{f \in V} \mathbb{E}\|\tilde{f}-f\|^{2},
$$

where $\|g\|$ is the $L^{2}$-norm of a function $g(\cdot)$ and the infimum is taken over all possible estimators $\tilde{f}(\cdot)$ (measurable functions taking their values in a set containing $V$ ) of $f(\cdot)$, based on observations from model (1.1)).

For $M=M_{n}$ and $N=n / M_{n}$, denote

$$
\tau_{\kappa}(m, n)=M^{-1} \sum_{l=1}^{M} N^{-2 \kappa d_{l}}\left|g_{m}\left(u_{l}\right)\right|^{2 \kappa}, \quad \kappa=1 \text { or } 2 \text { or } 4,
$$

and

$$
\Delta_{\kappa}(j, n)=\left|C_{j}\right|^{-1} \sum_{m \in C_{j}} \tau_{\kappa}(m, n)\left[\tau_{1}(m, n)\right]^{-2 \kappa}, \quad \kappa=1 \text { or } 2 .
$$

The expression $\tau_{1}(m, n)$ appears in both the lower and the upper bounds for the $L^{2}$-risk. Hence, we impose the following assumption:

Assumption A2: For some constants $\nu_{1}, \nu_{2}, \lambda_{1}, \lambda_{2} \in \mathbb{R}, \alpha_{1}, \alpha_{2} \geq 0\left(\lambda_{1}, \lambda_{2}>0\right.$ if $\left.\alpha_{1}=\alpha_{2}=0, \nu_{1}=\nu_{2}=0\right)$ and $K_{3}, K_{4}, \beta>0$, independent of $m$ and $n$, and for some sequence $\varepsilon_{n}>0$, independent of $m$, one has

$$
K_{3} \varepsilon_{n}|m|^{-2 \nu_{1}}(\ln |m|)^{-\lambda_{1}} e^{-\alpha_{1}|m|^{\beta}} \leq \tau_{1}(m, n) \leq K_{4} \varepsilon_{n}|m|^{-2 \nu_{2}}(\ln |m|)^{-\lambda_{2}} e^{-\alpha_{2}|m|^{\beta}},
$$


where either $\alpha_{1} \alpha_{2} \neq 0$ or $\alpha_{1}=\alpha_{2}=0$ and $\nu_{1}=\nu_{2}=\nu>0$. The sequence $\varepsilon_{n}$ in (4.5) is such that

$$
n^{*}=n \varepsilon_{n} \rightarrow \infty \quad(n \rightarrow \infty) .
$$

Under Assumptions A1 and A2, the following statement is true.

Theorem 1 Let Assumptions A1 and A2 hold. Let $\left\{\phi_{j_{0}, k}(\cdot), \psi_{j, k}(\cdot)\right\}$ be the periodic Meyer wavelet basis discussed in Section 3. Let $s>\max (0,1 / p-1 / 2), 1 \leq p \leq \infty, 1 \leq q \leq \infty$ and $A>0$. Then, as $n \rightarrow \infty$,

$$
R_{n}\left(B_{p, q}^{s}(A)\right) \geq \begin{cases}C\left(n^{*}\right)^{-\frac{2 s}{2 s+2 \nu+1}}\left(\ln n^{*}\right)^{\frac{2 s \lambda_{2}}{2 s+2 \nu+1}}, & \text { if } \alpha_{1}=\alpha_{2}=0, \nu(2-p)<p s^{*} \\ C\left(\frac{\ln n^{*}}{n^{*}}\right)^{\frac{2 s^{*}}{2 s^{*}+2 \nu}}\left(\ln n^{*}\right)^{\frac{2 s^{*} \lambda_{2}}{2 s^{*}+2 \nu},}, & \text { if } \alpha_{1}=\alpha_{2}=0, \nu(2-p) \geq p s^{*} \\ C\left(\ln n^{*}\right)^{-\frac{2 s^{*}}{\beta}}, & \text { if } \alpha_{1} \alpha_{2} \neq 0 .\end{cases}
$$

\section{$5 \quad$ Minimax Upper Bounds for the $L^{2}$-Risk}

Let $\hat{f}_{n}(\cdot)$ be the (block thresholding) wavelet estimator defined by (3.9). Choose now $j_{0}$ and $J$ such that

$$
\begin{array}{r}
2^{j_{0}}=\ln n^{*}, \quad 2^{J}=\left(n^{*}\right)^{\frac{1}{2 \nu+1}}, \quad \text { if } \quad \alpha_{1}=\alpha_{2}=0, \\
2^{j_{0}}=\frac{3}{8 \pi}\left(\frac{\ln n^{*}}{2 \alpha}\right)^{\frac{1}{\beta}}, 2^{J}=2^{j_{0}}, \quad \text { if } \quad \alpha_{1} \alpha>0 .
\end{array}
$$

(Since $j_{0}>J-1$ when $\alpha_{1} \alpha>0$, the estimator (3.9) only consists of the first (linear) part and, hence, $\lambda_{j}$ does not need to be selected in this case.) Set, for some constant $\mu>0$, large enough,

$$
\lambda_{j}=\mu^{2}\left(n^{*}\right)^{-1} \ln \left(n^{*}\right) 2^{2 \nu j} j^{\lambda_{1}}, \quad \text { if } \quad \alpha_{1}=\alpha_{2}=0 .
$$

Note that the choices of $j_{0}, J$ and $\lambda_{j}$ are independent of the parameters, $s, p, q$ and $A$ of the Besov ball $B_{p, q}^{s}(A)$; hence, the estimator (3.9) is adaptive with respect to these parameters.

Denote $(x)_{+}=\max (0, x)$

$$
\varrho= \begin{cases}\frac{(2 \nu+1)(2-p)_{+}}{p(2 s+2 \nu+1)}, & \text { if } \nu(2-p)<p s^{*}, \\ \frac{(q-p)_{+}}{q}, & \text { if } \nu(2-p)=p s^{*} \\ 0, & \text { if } \nu(2-p)>p s^{*}\end{cases}
$$

Assume that, in the case of $\alpha_{1}=\alpha_{2}=0$, the sequence $\varepsilon_{n}$ is such that

$$
-h_{1} \ln n \leq \ln \left(\varepsilon_{n}\right) \leq h_{2} \ln n
$$

for some constants $h_{1}, h_{2} \in(0,1)$. Observe that condition (5.5) implies (4.6) and that $\ln n^{*} \asymp \ln n(n \rightarrow \infty)$. (Here, and in what follows, $u(n) \asymp v(n)$ means that there exist 
constants $C_{1}, C_{2}\left(0<C_{1} \leq C_{2}<\infty\right)$, independent of $n$, such that $0<C_{1} v(n) \leq u(n) \leq$ $C_{2} v(n)<\infty$ for $n$ large enough.)

The proof of the minimax upper bounds for the $L^{2}$-risk is based on the following two lemmas.

Lemma 2 Let Assumptions A1 and A2 hold. Let the estimators $\widehat{a}_{j_{0} k}$ and $\widehat{b}_{j k}$ of the scaling and wavelet coefficients $a_{j_{0} k}$ and $b_{j k}$, respectively, be given by (3.6) with $\widehat{f}_{m}$ defined by (3.4). Then, for all $j \geq j_{0}$,

$$
\mathbb{E}\left|\widehat{a}_{j_{0} k}-a_{j_{0} k}\right|^{2} \leq C n^{-1} \Delta_{1}\left(j_{0}, n\right) \quad \text { and } \quad \mathbb{E}\left|\widehat{b}_{j k}-b_{j k}\right|^{2} \leq C n^{-1} \Delta_{1}(j, n) .
$$

If $\alpha_{1}=\alpha_{2}=0$ and (5.5) holds, then, for any $j \geq j_{0}$,

$$
\mathbb{E}\left|\widehat{b}_{j k}-b_{j k}\right|^{4} \leq C n^{3}(\ln n)^{3 \lambda_{1}}\left(n^{*}\right)^{-\frac{3}{2 \nu+1}} .
$$

Lemma 3 Let Assumptions A1, A2 and (5.5) hold. Let the estimators $\widehat{b}_{j k}$ of the wavelet coefficients $b_{j k}$ be given by (3.6) with $\widehat{f}_{m}$ defined by (3.4). Let

$$
\mu \geq \sqrt{\frac{2}{1-h_{1}}}\left[\sqrt{c_{1}}+\frac{\sqrt{8 \pi \kappa}}{\sqrt{K_{3}}}(\ln 2)^{\lambda_{1} / 2}\left(\frac{2 \pi}{3}\right)^{\nu}\right],
$$

where $c_{1}, K_{3}$ and $h_{1}$ are defined in (8.8), (4.5) and (5.5), respectively. Then, for all $j \geq j_{0}$ and any $\kappa>0$,

$$
\mathbb{P}\left(\sum_{k \in U_{j r}}\left|\widehat{b}_{j k}-b_{j k}\right|^{2} \geq\left(4 n^{*}\right)^{-1} \mu^{2} 2^{2 \nu j} j^{\lambda} \ln n^{*}\right) \leq n^{-\kappa} .
$$

Under Assumptions A1 and A2, and using Lemmas 2 and 3, the following statement is true.

Theorem 2 Let Assumptions A1 and A2 hold. Let $\hat{f}_{n}(\cdot)$ be the wavelet estimator defined by (3.9), with $j_{0}$ and $J$ given by (5.1) (if $\alpha_{1}=\alpha_{2}=0$ ) or (5.2) (if $\alpha_{1} \alpha_{2}>0$ ) and $\mu$ satisfying (5.8) with $\kappa=5$. Let $s>1 / p^{\prime}, 1 \leq p \leq \infty, 1 \leq q \leq \infty$ and $A>0$. Then, under (4.6) if $\alpha_{1} \alpha_{2}>0$ or (5.5) if $\alpha_{1}=\alpha_{2}=0$, as $n \rightarrow \infty$,

$\sup _{f \in B_{p, q}^{s}(A)} \mathbb{E}\left\|\hat{f}_{n}-f\right\|^{2} \leq \begin{cases}C\left(n^{*}\right)^{-\frac{2 s}{2 s+2 \nu+1}}(\ln n)^{\varrho+\frac{2 s \lambda_{1}}{2 s+2 \nu+1}}, & \text { if } \alpha_{1}=\alpha_{2}=0, \nu(2-p)<p s^{*}, \\ C\left(\frac{\ln n}{n^{*}}\right)^{\frac{2 s^{*}}{2 s^{*}+2 \nu}}(\ln n)^{\varrho+\frac{2 s^{*} \lambda_{1}}{2 s^{*}+2 \nu}}, & \text { if } \quad \alpha_{1}=\alpha_{2}=0, \nu(2-p) \geq p s^{*}, \\ C\left(\ln n^{*}\right)^{-\frac{2 s^{*}}{\beta}}, & \text { if } \quad \alpha_{1} \alpha_{2}>0 .\end{cases}$ 
Remark 2 Theorems 1 and 2 implies that, for the $L^{2}$-risk, the wavelet estimator $\hat{f}_{n}(\cdot)$ defined by (3.9) is asymptotical optimal (in the minimax sense), or near optimal within a logarithmic factor, over a wide range of Besov balls $B_{p, q}^{s}(A)$ of radius $A>0$ with $s>\max (1 / p, 1 / 2), 1 \leq p \leq \infty$ and $1 \leq q \leq \infty$. The convergence rates depend on the balance between the smoothness parameter $s$ (of the response function $f(\cdot)$ ), the kernel parameters $\nu, \beta, \lambda_{1}$ and $\lambda_{2}$ (of the blurring function $g(\cdot, \cdot)$ ), the long memory parameters $d_{l}, l=1,2 \ldots, M$ (of the error sequence $\boldsymbol{\xi}^{(l)}$ ), and how the total number of observations $n$ is distributed among the total number of channels $M$. In particular, $M$ and $d_{l}, l=1,2, \ldots, M$, jointly determine the value of $\varepsilon_{n}$ which, in turn, defines the "essential" convergence rate $n^{*}=n \varepsilon_{n}$ which may differ considerably from $n$. For example, if $M=M_{n}=n^{\theta}, 0 \leq \theta<1$ and $\left|g_{m}\left(u_{l}\right)\right|^{2} \asymp|m|^{-2 \nu}$ for every $l=1,2 \ldots, M$, then

$$
\varepsilon_{n}=M^{-1} \sum_{l=1}^{M} N^{-2 d_{l}},
$$

and, therefore, $n^{1-2 d^{*}(1-\theta)} \leq n^{*} \leq n$, where $d^{*}=\max _{1 \leq l \leq M} d_{l}$, so that, $n^{*}$ can take any value between $n^{1-2 d^{*}(1-\theta)}$ and $n$. This is further illustrated in Section 6 below.

\section{$6 \quad$ Illustrative Examples}

In this section, we consider some illustrative examples of application of the theory developed in the previous sections. They are particular examples of inverse problems in mathematical physics where one needs to recover initial or boundary conditions on the basis of observations from a noisy solution of a partial differential equation.

We assume that condition (2.4) holds true and that there exist $\theta_{1}$ and $\theta_{2}$, such that $M=M_{n}$ satisfies

$$
n^{\theta_{1}} \leq M \leq n^{\theta_{2}}, \quad 0 \leq \theta_{1} \leq \theta_{2}<1 .
$$

(Note that, under (6.1), $n^{1-\theta_{2}} \leq N \leq n^{1-\theta_{1}}$.)

Example 1 Consider the case when $g_{m}(\cdot), m=0, \pm 1, \pm 2, \ldots$, is of the form

$$
g_{m}(u)=C_{g} \exp \left(-K|m|^{\beta} q(u)\right), \quad u \in U,
$$

where $q(\cdot)$ in (6.2) is such that, for some $q_{1}$ and $q_{2}$,

$$
0<q_{1} \leq q(u) \leq q_{2}<\infty, \quad u \in U .
$$

This set up takes place in the estimation of the initial condition in the heat conductivity equation or the estimation of the boundary condition for the Dirichlet problem of the Laplacian on the unit circle (see Examples 1 and 2 of Pensky and Sapatinas (2009, 2010)). In the former case, $g_{m}(u)=\exp \left(-4 \pi^{2} m^{2} u\right), u \in U$, so that $K=4 \pi^{2}, \beta=2$, $q(u)=u, q_{1}=a$ and $q_{2}=b$. In the latter case, $g_{m}(u)=C u^{|m|}=C \exp (-|m| \ln (1 / u))$, 
$0<r_{1} \leq u \leq r_{2}<1$, so that $K=1, \beta=1, q(u)=\ln (1 / u), q_{1}=\ln \left(1 / r_{2}\right)$ and $q_{2}=\ln \left(1 / r_{1}\right)$.

It is easy to see that, under conditions (6.2) and (‥3), for $\tau_{1}(m, n)$ given in (4.3),

$$
\tau_{1}(m, n) \leq C_{g} \varepsilon_{n} \exp \left(-2 K q_{1}|m|^{\beta}\right) \quad \text { and } \quad \tau_{1}(m, n) \geq C_{g} \varepsilon_{n} \exp \left(-2 K q_{2}|m|^{\beta}\right),
$$

where $\varepsilon_{n}$ is of the form (5.11). Assumptions (2.4) and (6.1) lead to the following bounds for $n^{*}$ :

$$
n^{1-2 d^{*}\left(1-\theta_{1}\right)} \leq n^{*} \leq n,
$$

so that $\ln n \asymp \ln n^{*}$. Therefore, according to Theorems 1 and 2 ,

$$
R_{n}\left(B_{p, q}^{s}(A)\right) \asymp(\ln n)^{-\frac{2 s^{*}}{\beta}} .
$$

Note that, in this case, the value of $d^{*}$ has absolutely no bearing on the convergence rates of the linear wavelet estimators: the convergence rates are determined entirely by the properties of the smoothness parameter $s^{*}$ (of the response function $f(\cdot)$ ) and the kernel parameter $\beta$ (of the blurring function $g(\cdot, \cdot)$ ).

In other words, in case of super-smooth convolutions, LRD does not influence the convergence rates of the suugested wavelet estimator. A similar effect is observed in the case of kernel smoothing, see Section 2.2 in Kulik (2008).

Example 2 Suppose that the blurring function $g(\cdot, \cdot)$ is of a box-car like kernel, i.e.,

$$
g(u, t)=0.5 q(u) \mathbb{I}(|t|<u), \quad u \in U, \quad t \in T,
$$

where $q(\cdot)$ is some positive function which satisfies conditions (6.3). In this case, the functional Fourier coefficients $g_{m}(\cdot)$ are of the form

$$
g_{0}(u)=1 \quad \text { and } \quad g_{m}(u)=(2 \pi m)^{-1} \gamma(u) \sin (2 \pi m u), \quad m \in \mathbb{Z} \backslash\{0\}, \quad u \in U .
$$

It is easy to see that estimation of the initial speed of a wave on a finite interval (see Example 4 of Pensky and Sapatinas (2009) or Example 3 of Pensky and Sapatinas (2010)) leads to $g_{m}(\cdot)$ of the form (6.6) with $q(u)=1$. Assume, without loss of generality, that $u \in[0,1]$, so that $a=0, b=1$, and consider (equispaced channels) $u_{l}=l / M$, $l=1,2, \ldots, M$, such that

$$
d_{l}=a_{1} u_{l}+a_{2}, \quad 0 \leq a_{2} \leq d^{*}<1 / 2,0 \leq a_{1}+a_{2} \leq d^{*}<1 / 2,
$$

i.e., condition (2.4) holds. Note that if $a_{1}=0$, then

$$
\tau_{1}(m, n) \asymp M^{-1} N^{-2 a_{2}}\left(4 \pi^{2} m^{2}\right)^{-1} \sum_{l=1}^{M} \sin ^{2}(2 \pi m l / M),
$$


which is similar to the expression for $\tau_{1}(m, n)$ studied in Section 6 of Pensky and Sapatinas (2010). Following their calculations, one obtains that, if $j_{0}$ in (3.9) is such that $2^{j_{0}}>(\ln n)^{\delta}$ for some $\delta>0$ and $M \geq(32 \pi / 3) n^{1 / 3}$, then, for $n$ and $|m|$ large enough,

$$
\tau_{1}(m, n) \asymp N^{-2 a_{2}} m^{-2} .
$$

Assume now, without loss of generality, that $a_{1} \geq 0$. (Note that the case of $a_{1} \leq 0$ can be handled similarly by changing $u$ to $1-u$.) Below, we shall show that, in this case, a similar result can be obtained under less stringent conditions on $M=M_{n}$. Indeed, the following statement is true.

Lemma 4 Let $g(\cdot, \cdot)$ be of the form (6.5), where $q(\cdot)$ is some positive function which satisfies (6.3), and let $d_{l}, l=1,2, \ldots, M$, be given by (6.7) with $a_{1} \geq 0$. Assume (without loss of generality) that $U=[0,1]$, and consider $u_{l}=l / M, l=1,2, \ldots, M$. Let $M=M_{n}$ satisfy (6.1) with $\theta_{1}>0$ if $a_{1}>0$ and $M \geq(32 \pi / 3) n^{1 / 3}$ if $a_{1}=0$. If $m \in A_{j}$, where $\left|A_{j}\right|=C_{m} 2^{j}$, for some absolute constant $C_{m}>0$, with $j \geq j_{0}>0$, where $j_{0}$ is such that $2^{j_{0}} \geq C_{0} \ln n$ for some $C_{0}>0$, then, for $n$ and $|m|$ large enough,

$$
\tau_{1}(m, n) \asymp N^{-2 a_{2}} m^{-2}(\log n)^{-1} .
$$

It follows immediately from Lemma 4 that, if

$$
M=M_{n}=n^{\theta}, \quad 0<\theta<1,
$$

then Assumption A2 holds with $\alpha_{1}=\alpha_{2}=0, \nu_{1}=\nu_{2}=\nu=2, \varepsilon_{n}=n^{-2 a_{2}(1-\theta)}(\ln n)^{-1}$ and $\lambda_{1}=\lambda_{2}=0$. Note that $\varepsilon_{n}$ satisfies conditions (4.6) and (5.5), so that $\ln n \asymp \ln n^{*}$. Therefore, according to Theorems 1 and 2 ,

$$
R_{n}\left(B_{p, q}^{s}(A)\right) \geq\left\{\begin{array}{lll}
C\left(n^{*}\right)^{-\frac{2 s}{2 s+5}}, & \text { if } & 4-2 p<p s^{*} \\
C\left(\frac{\ln n^{*}}{n^{*}}\right)^{\frac{s^{*}}{s^{*}+2}}, & \text { if } & 4-2 p \geq p s^{*}
\end{array}\right.
$$

and

$$
\sup _{f \in B_{p, q}^{s}(A)} \mathbb{E}\left\|\hat{f}_{n}-f\right\|^{2} \leq\left\{\begin{array}{lll}
C\left(n^{*}\right)^{-\frac{2 s}{2 s+5}}(\ln n)^{\varrho}, & \text { if } & 4-2 p<p s^{*} \\
C\left(\frac{\ln n}{n^{*}}\right)^{\frac{s^{*}}{s^{*}+2}}(\ln n)^{\varrho}, & \text { if } & 4-2 p \geq p s^{*}
\end{array}\right.
$$

where

$$
n^{*}=n^{1-2 a_{2}(1-\theta)}(\ln n)^{-1}
$$

and

$$
\varrho=\left\{\begin{array}{lll}
\frac{\left(5(2-p)_{+}\right.}{p(2 s+5)}, & \text { if } 4-2 p<p s^{*} \\
\frac{(q-p)_{+}}{q}, & \text { if } 4-2 p=p s^{*} \\
0, & \text { if } 4-2 p>p s^{*}
\end{array}\right.
$$

Note that LRD affects the convergence rates in this case via the parameter $a_{2}$ that appears in the definition (6.7). 


\section{Discussion.}

Deconvolution is the common problem in many areas of signal and image processing which include, for instance, LIDAR (Light Detection and Ranging) remote sensing and reconstruction of blurred images. LIDAR is a laser device which emits pulses, reflections of which are gathered by a telescope aligned with the laser (see, e.g., Park, Dho \& Kong (1997) and Harsdorf \& Reuter (2000)). The return signal is used to determine distance and the position of the reflecting material. However, if the system response function of the LIDAR is longer than the time resolution interval, then the measured LIDAR signal is blurred and the effective accuracy of the LIDAR decreases. If $M(M \geq 2)$ LIDAR devices are used to recover a signal, then we talk about a multichannel deconvolution problem. This leads to the discrete model (1.1) considered in this work.

The multichannel deconvolution model (1.1) can also be thought of as the discrete version of a model referred to as the functional deconvolution model by Pensky and Sapatinas $(2009,2010)$. The functional deconvolution model has a multitude of applications. In particular, it can be used in a number of inverse problems in mathematical physics where one needs to recover initial or boundary conditions on the basis of observations from a noisy solution of a partial differential equation. Lattes \& Lions (1967) initiated research in the problem of recovering the initial condition for parabolic equations based on observations in a fixed-time strip. This problem and the problem of recovering the boundary condition for elliptic equations based on observations in an internal domain were studied in Golubev \& Khasminskii (1999); the latter problem was also discussed in Golubev (2004). Some of these specific models were considered in Section 6 .

The multichannel deconvolution model (1.1) and its continuous version, the functional deconvolution model, were studied by Pensky and Sapatinas (2009, 2010), under the assumption that errors are independent and identically distributed Gaussian random variables. The objective of this work was to study the multichannel deconvolution model (1.1) from a minimax point of view, with the relaxation that errors exhibit LRD. We were not limited our consideration to a specific type of LRD: the only restriction made was that the errors should satisfy a general assumption in terms of the smallest and larger eigenvalues of their covariance matrices. In particular, minimax lower bounds for the $L^{2}$-risk in model (1.1) under such assumption were derived when $f(\cdot)$ is assumed to belong to a Besov ball and $g(\cdot, \cdot)$ has smoothness properties similar to those in Pensky and Sapatinas $(2009,2010)$, including both regular-smooth and super-smooth convolutions. In addition, an adaptive wavelet estimator of $f(\cdot)$ was constructed and shown that such estimator is asymptotically optimal (in the minimax sense), or near-optimal within a logarithmic factor, in a wide range of Besov balls. The convergence rates of the resulting estimators depend on the balance between the smoothness parameter (of the response function $f(\cdot)$ ), the kernel parameters (of the blurring function $g(\cdot, \cdot)$ ), and the long memory parameters

$d_{l}, l=1,2 \ldots, M$ (of the error sequence $\boldsymbol{\xi}^{(l)}$ ), and how the total number of observations is distributed among the total number of channels. Note that SRD is implicitly included in our results by selecting $d_{l}=0, l=1,2, \ldots, M$. In this case, the convergence rates 
we obtained coincide with the convergence rates obtained under the assumption of independent and identically distributed Gaussian errors by Pensky and Sapatinas (2009, 2010).

Under the assumption that the errors are independent and identically distributed Gaussian random variables, for box-car kernels, it is known that, when the number of channels in the multichannel deconvolution model (1.1) is finite, the precision of reconstruction of the response function increases as the number of channels $M$ grow (even when the total number of observations $n$ for all channels $M$ remains constant) and this requires the channels to form a Badly Approximable (BA) $M$-tuple (see De Canditiis and Pensky (2004, 2007)). Under the same assumption for the errors, Pensky and Sapatinas (2009, 2010) showed that the construction of a BA $M$-tuple for the channels is not needed and a uniform sampling strategy for the channels with the number of channels increasing at a polynomial rate (i.e., $u_{l}=l / M, l=1,2, \ldots, M$, for $M=M_{n} \geq(32 \pi / 3) n^{1 / 3}$ ) suffices to construct an adaptive wavelet estimator that is asymptotically optimal (in the minimax sense), or near-optimal within a logarithmic factor, in a wide range of Besov balls, when the blurring function $g(\cdot, \cdot)$ is of box-car like kernel (including both the standard box-car kernel and the kernel that appears the estimation of the initial speed of a wave on a finite interval). Example 2 showed that a similar result is still possible under long-range dependence with (equispaced channels) $u_{l}=l / M, l=1,2, \ldots, M, n^{\theta_{1}} \leq M=M_{n} \leq n^{\theta_{2}}$, for some $0 \leq \theta_{1} \leq \theta_{2}<1$ when $d_{l}=a_{1} u_{l}+a_{2}, l=1,2, \ldots, M, 0 \leq a_{2}<1 / 2,0 \leq a_{1}+a_{2}<1 / 2$.

However, in real-life situations, the number of channels $M=M_{n}$ usually refers to the number of physical devices and, consequently, may grow to infinity only at a slow rate as $n \rightarrow \infty$. When $M=M_{n}$ grows slowly as $n$ increases, (i.e., $M=M_{n}=o\left((\ln n)^{\alpha}\right)$ for some $\alpha \geq 1 / 2$ ), in the multichannel deconvolution model with independent and identically distributed Gaussian errors, Pensky and Sapatinas (2011) developed a procedure for the construction of a BA $M$-tuple on a specified interval, of a non-asymptotic length, together with a lower bound associated with this $M$-tuple, which explicitly shows its dependence on $M$ as $M$ is growing. This result was further used for the derivation of upper bounds for the $L^{2}$-risk of the suggested adaptive wavelet thresholding estimator of the unknown response function and, furthermore, for the choice of the optimal number of channels $M$ which minimizes the $L^{2}$-risk. It would be of interest to see whether or not similar upper bounds are possible under long-range dependence. Another avenue of possible research is to consider an analogous minimax study for the functional deconvolution model (i.e., the continuous version of the multichannel deconvolution model (1.1)) under long rangedependence (e.g., modeling the errors as a fractional Brownian motion) and examine the effect of the convergence rates between the two models, similar to the convergence rate study of Pensky and Sapatinas (2010) when the errors were considered to be independent and identically distributed Gaussian random variables.

\section{Proofs}

\subsection{Proofs of the Statements in Section 2}

Proof of Lemma 1. We prove the upper bound only since the proof of the lower bound 
is similar. By (2.1)-(2.2), and the definitions of $\mathcal{H}_{N}$ and $\mathcal{F}_{d}$,

$$
\lambda_{\max }(\boldsymbol{\Sigma}) \leq C_{\max } \sup _{h \in \mathcal{H}_{N}} \int_{-\pi}^{\pi} h(\lambda)|\lambda|^{-2 d} d \lambda=2 C_{\max } \sup _{h \in \mathcal{H}_{N}} \int_{0}^{\pi} h(\lambda)|\lambda|^{-2 d} d \lambda .
$$

Now, we split $\int_{0}^{\pi}=\int_{0}^{\pi / N}+\int_{\pi / N}^{\pi}$. Since $d<1 / 2$, for the first integral, we have

$$
\int_{0}^{\pi / N} h(\lambda)|\lambda|^{-2 d} d \lambda \leq N \int_{0}^{\pi / N} \lambda^{-2 d} d \lambda=N \frac{1}{1-2 d}\left(\frac{\pi}{N}\right)^{-2 d+1}=\frac{1}{1-2 d} N^{2 d} .
$$

For the second integral, since $d \geq 0$, we have

$$
\int_{\pi / N}^{\pi} h(\lambda)|\lambda|^{-2 d} d \lambda \leq\left(\frac{\pi}{N}\right)^{-2 d} \int_{\pi / N}^{\pi} h(\lambda) d \lambda \leq\left(\frac{\pi}{N}\right)^{-2 d} \int_{0}^{\pi} h(\lambda) d \lambda \leq \pi(2 \pi)^{-2 d} N^{2 d} .
$$

This completes the proof of the lemma.

\subsection{Proof of the Minimax Lower Bounds for the $L^{2}$-Risk}

In order to prove Theorem 1, we consider two cases: the dense case and the sparse case, when the hardest functions to estimate are, respectively, uniformly spread over the unit interval $T$ and are represented by only one term in a wavelet expansion.

The proof of Theorem 1 is based on Lemma A.1 of Bunea, Tsybakov and Wegkamp (2007), which we reformulate here for the case of the $L^{2}$-risk.

Lemma 5 [Bunea, Tsybakov, Wegkamp (2007), Lemma A.1] Let $\Omega$ be a set of functions of cardinality $\operatorname{card}(\Omega) \geq 2$, such that

(i) $\|f-g\|^{2} \geq 4 \delta^{2}$ for $f, g \in \Omega, \quad f \neq g$,

(ii) the Kullback divergences $K\left(P_{f}, P_{g}\right)$ between the measures $P_{f}$ and $P_{g}$ satisfy the inequality $K\left(P_{f}, P_{g}\right) \leq \log (\operatorname{card}(\Omega)) / 16$ for $f, g \in \Omega$.

Then, for some absolute constant $C>0$, one has

$$
\inf _{T_{n}} \sup _{f \in \Omega} \mathbb{E}_{f}\left\|T_{n}-f\right\|^{2} \geq C \delta^{2} .
$$

The dense case. Let $\boldsymbol{\omega}$ be the vector with components $\omega_{k}=\{0,1\}$. Denote the set of all possible vectors $\boldsymbol{\omega}$ by $\Omega=\left\{(0,1)^{2^{j}}\right\}$. Note that the vector $\boldsymbol{\omega}$ has $\aleph=2^{j}$ entries and, hence, $\operatorname{card}(\Omega)=2^{\aleph}$. Let $H(\tilde{\boldsymbol{\omega}}, \boldsymbol{\omega})=\sum_{k=0}^{2^{j}-1} \mathbb{I}\left(\tilde{\boldsymbol{\omega}}_{k} \neq \omega_{k}\right)$ be the Hamming distance between the binary sequences $\boldsymbol{\omega}$ and $\tilde{\boldsymbol{\omega}}$. Then, the Varshamov-Gilbert Lemma (see, e.g., Tsybakov (2008), p. 104) states that one can choose a subset $\Omega_{1}$ of $\Omega$, of cardinality at least $2^{\aleph / 8}$, such that $H(\tilde{\boldsymbol{\omega}}, \boldsymbol{\omega}) \geq \aleph / 8$ for any $\boldsymbol{\omega}, \tilde{\boldsymbol{\omega}} \in \Omega_{1}$.

Consider two arbitrary sequences $\boldsymbol{\omega}, \tilde{\boldsymbol{\omega}} \in \Omega_{1}$ and the functions $f_{j}$ and $\tilde{f}_{j}$ given by

$$
f_{j}(t)=\rho_{j} \sum_{k=0}^{2^{j}-1} \omega_{k} \psi_{j k}(t) \quad \text { and } \quad \tilde{f}_{j}(t)=\rho_{j} \sum_{k=0}^{2^{j}-1} \tilde{\omega}_{k} \psi_{j k}(t), \quad t \in T .
$$


Choose $\rho_{j}=A 2^{-j(s+1 / 2)}$, so that $f_{j}, \tilde{f}_{j} \in B_{p, q}^{s}(A)$. Then, calculating the $L^{2}$-norm difference of $f_{j}$ and $\tilde{f}_{j}$, we obtain

$$
\left\|\tilde{f}_{j}-f_{j}\right\|^{2}=\rho_{j}^{2}\left\|\sum_{k=0}^{2^{j}-1}\left(\tilde{\omega}_{k}-\omega_{k}\right) \psi_{j k}\right\|^{2}=\rho_{j}^{2} H(\tilde{\boldsymbol{\omega}}, \boldsymbol{\omega}) \geq 2^{j} \rho_{j}^{2} / 8 .
$$

Hence, we get $4 \delta^{2}=2^{j} \rho_{j}^{2} / 8$ in condition $(i)$ of Lemma 5 ,

In order to apply Lemma 5, one needs to also verify condition $(i i)$. For $f_{\boldsymbol{\omega}}$ with $\boldsymbol{\omega} \in \Omega$, denote by $\mathbf{h}_{l, \boldsymbol{\omega}}$ and $\mathbf{h}_{l, \tilde{\boldsymbol{\omega}}}$, the vectors with components, respectively,

$$
\begin{aligned}
& h_{\boldsymbol{\omega}}\left(u_{l}, t_{i}\right)=g\left(u_{l}, t_{i}-\cdot\right) * f_{\boldsymbol{\omega}}(\cdot), \quad i=1,2, \ldots, N, \\
& h_{\tilde{\boldsymbol{\omega}}}\left(u_{l}, t_{i}\right)=g\left(u_{l}, t_{i}-\cdot\right) * f_{\tilde{\boldsymbol{\omega}}}(\cdot), \quad i=1,2, \ldots, N \text {. }
\end{aligned}
$$

Then,

$$
\begin{aligned}
K\left(P_{\boldsymbol{\omega}}, P_{\tilde{\boldsymbol{\omega}}}\right) & =0.5 \sum_{l=1}^{M}\left(\mathbf{h}_{l, \boldsymbol{\omega}}-\mathbf{h}_{l, \tilde{\boldsymbol{\omega}}}\right)^{T}\left(\boldsymbol{\Sigma}^{(l)}\right)^{-1}\left(\mathbf{h}_{l, \boldsymbol{\omega}}-\mathbf{h}_{l, \tilde{\boldsymbol{\omega}}}\right) \\
& \leq 0.5 \sum_{l=1}^{M} \lambda_{\max }\left(\left(\boldsymbol{\Sigma}^{(l)}\right)^{-1}\right)\left\|\mathbf{h}_{l, \boldsymbol{\omega}}-\mathbf{h}_{l, \tilde{\boldsymbol{\omega}}}\right\|^{2} .
\end{aligned}
$$

Now, since $\boldsymbol{\omega}$ and $\tilde{\boldsymbol{\omega}}$ are binary vectors, using Plancherel's formula, (8.1), and the fact that $\left|\psi_{j k, m}\right| \leq 2^{-j / 2}$, we derive that, under Assumptions A1 and A2,

$$
\begin{aligned}
K\left(P \boldsymbol{\omega}, P_{\tilde{\boldsymbol{\omega}}}\right) & \leq 0.5 N M \rho_{j}^{2} \sum_{m \in C_{j}} \frac{1}{M} \sum_{l=1}^{M}\left|g_{m}\left(u_{l}\right)\right|^{2} K_{1}^{-1} N^{-2 d_{l}} \\
& \leq 2 \pi K_{1}^{-1} n 2^{j} \rho_{j}^{2} \Delta_{1}(j, n) \leq 2 \pi A^{2} K_{1}^{-1} n 2^{-2 j s} \Delta_{1}(j, n),
\end{aligned}
$$

where $\Delta_{1}(j, n)$ is defined by (4.4).

Direct calculations yield that, under Assumptions A1, A2 and (4.6), for some constants $c_{3}>0$ and $c_{4}>0$, independent of $n$,

$$
\Delta_{1}(j, n) \leq \begin{cases}c_{3} \varepsilon_{n}^{-1} 2^{2 \nu j} j^{\lambda_{2}}, & \text { if } \alpha_{1}=\alpha_{2}=0, \\ c_{4} \varepsilon_{n}^{-1} 2^{2 \nu_{1} j} j^{\lambda_{2}} \exp \left\{\alpha_{1}\left(\frac{8 \pi}{3}\right)^{\beta} 2^{j \beta}\right\}, & \text { if } \alpha_{1} \alpha>0 .\end{cases}
$$

Apply now Lemma 5 with $j$ such that

$$
2 \pi A^{2} K_{1}^{-1} n 2^{-2 j s} \Delta_{1}(j, n) \leq 2^{j} \ln 2 / 16,
$$


i.e.,

$$
2^{j} \asymp \begin{cases}{\left[n^{*}\left(\ln n^{*}\right)^{-\lambda_{2}}\right]^{\frac{1}{2 s+2 \nu+1}},} & \text { if } \beta=0, \\ \left(\ln n^{*}\right)^{1 / \beta}, & \text { if } \beta>0,\end{cases}
$$

to obtain

$$
\delta^{2}= \begin{cases}{\left[n^{*}\left(\ln n^{*}\right)^{-\lambda_{2}}\right]^{-\frac{2 s}{2 s+2 \nu+1}},} & \text { if } \beta=0 \\ \left(\ln n^{*}\right)^{-2 s / \beta}, & \text { if } \beta>0 .\end{cases}
$$

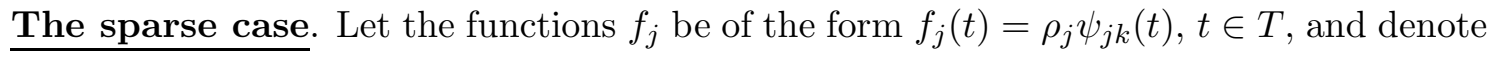

$$
\Omega=\left\{f_{j}(t)=\rho_{j} \psi_{j k}(t): k=0,1, \ldots, 2^{j}-1, f_{0}=0\right\} .
$$

Thus, $\operatorname{card}(\Omega)=2^{j}$. Choose now $\rho_{j}=A 2^{-j s^{\prime}}$, so that $f_{j} \in B_{p, q}^{s}(A)$. It is easy to check that, in this case, one has $4 \delta^{2}=\rho_{j}^{2}$ in Lemma 囵, and that

$$
K\left(P_{\omega}, P_{\tilde{\omega}}\right) \leq 2 \pi A^{2} K_{1}^{-1} n 2^{-2 j s^{\prime}} \Delta_{1}(j, n) .
$$

With

$$
2^{j} \asymp \begin{cases}{\left[n^{*}\left(\ln n^{*}\right)^{-\lambda_{2}-1}\right]^{\frac{1}{2 s^{\prime}+2 \nu}},} & \text { if } \beta=0, \\ \left(\ln n^{*}\right)^{1 / \beta}, & \text { if } \beta>0,\end{cases}
$$

we then obtain that $K\left(P_{\omega}, P_{\tilde{\omega}}\right) \leq 2 \pi A^{2} K_{1}^{-1} n 2^{-2 j s^{\prime}} \Delta_{1}(j, n)$ and

$$
\delta^{2}= \begin{cases}{\left[\frac{n^{*}}{\left(\ln n^{*}\right)_{2}+1}\right]^{-\frac{2 s^{\prime}}{2 s^{\prime}+2 \nu}},} & \text { if } \beta=0, \\ \left(\ln n^{*}\right)^{-2 s^{\prime} / \beta}, & \text { if } \beta>0 .\end{cases}
$$

Recall that $s^{*}=\min \left\{s, s^{\prime}\right\}$. By noting that

$$
2 s /(2 s+2 \nu+1) \leq 2 s^{*} /\left(2 s^{*}+2 \nu\right), \quad \text { if } \quad \nu(2-p) \leq p s^{*},
$$

we then choose the highest of the lower bounds in (8.2) and (8.3). This completes the proof of the theorem.

\subsection{Proof of the Minimax Upper Bounds for the $L^{2}$-Risk.}

We start with proofs of Lemmas 2 and 3 ,

Proof of Lemma 2. First, consider model (1.1). Then, using (3.2), (3.4), (3.6) and (3.7), one has

$$
\widehat{a}_{j_{0} k}-a_{j_{0} k}=\sum_{m \in C_{j_{0}}}\left(\widehat{f}_{m}-f_{m}\right) \overline{\varphi_{m j_{0} k}}, \quad \widehat{b}_{j k}-b_{j k}=\sum_{m \in C_{j}}\left(\widehat{f}_{m}-f_{m}\right) \overline{\psi_{m j k}},
$$

where

$$
\widehat{f}_{m}-f_{m}=\frac{1}{\sqrt{N}}\left(\sum_{l=1}^{M} N^{-2 d_{l}} \overline{g_{m}\left(u_{l}\right)} z_{l m}\right) /\left(\sum_{l=1}^{M} N^{-2 d_{l}}\left|g_{m}\left(u_{l}\right)\right|^{2}\right) .
$$


Consider vector $\mathbf{V}^{(l)}$ with components

$$
V_{m}^{(l)}=N^{-2 d_{l}} \psi_{m j k} g_{m}\left(u_{l}\right)\left[\sum_{l=1}^{M} N^{-2 d_{l}}\left|g_{m}\left(u_{l}\right)\right|^{2}\right]^{-1} .
$$

It is easy to see that, due to $\left|\psi_{m j k}\right| \leq 2^{-j / 2}$ and the definition of $C_{j}$,

$$
\begin{aligned}
\left\|\mathbf{V}^{(l)}\right\|^{2} & =N^{-4 d_{l}} \sum_{m \in C_{j}}\left|\psi_{m j k}\right|^{2}\left|g_{m}\left(u_{l}\right)\right|^{2}\left[\sum_{l=1}^{M} N^{-2 d_{l}}\left|g_{m}\left(u_{l}\right)\right|^{2}\right]^{-2} \\
& \leq 4 \pi\left|C_{j}\right|^{-1} N^{-4 d_{l}} \sum_{m \in C_{j}}\left|g_{m}\left(u_{l}\right)\right|^{2}\left[\sum_{l=1}^{M} N^{-2 d_{l}}\left|g_{m}\left(u_{l}\right)\right|^{2}\right]^{-2} .
\end{aligned}
$$

Define

$$
v_{m}=\sum_{l=1}^{M} N^{-2 d_{l}}\left|g_{m}\left(u_{l}\right)\right|^{2}=M \tau_{1}(m, n) .
$$

Hence,

$$
\left\|\mathbf{V}^{(l)}\right\|^{2} \leq 4 \pi\left|C_{j}\right|^{-1} N^{-2 d_{l}} N^{-2 d_{l}} \sum_{m \in C_{j}}\left|g_{m}\left(u_{l}\right)\right|^{2} v_{m}^{-2} .
$$

Using Assumption A1, since $z_{l m}$ are independent for different $l^{\prime}$ s, we obtain

$$
\begin{aligned}
\mathbb{E}\left|\widehat{b}_{j k}-b_{j k}\right|^{2} & =\frac{1}{N} \sum_{m_{1}, m_{2} \in C_{j}} \bar{\psi}_{m_{1} j k} \psi_{m_{2} j k} \sum_{l=1}^{M} N^{-4 d_{l}} v_{m_{1}}^{-1} v_{m_{2}}^{-1} \overline{g_{m_{1}}\left(u_{l}\right)} g_{m_{2}}\left(u_{l}\right) \operatorname{Cov}\left(z_{l m_{1}}, \bar{z}_{l m_{2}}\right) \\
& =\frac{1}{N} \sum_{l=1}^{M} \overline{\mathbf{V}^{(l)}} \boldsymbol{\Sigma}^{(l)} \mathbf{V}^{(l)} \\
& \leq \frac{1}{N} \sum_{l=1}^{M} \lambda_{\max }\left(\boldsymbol{\Sigma}^{(l)}\right)\left\|\mathbf{V}^{(l)}\right\|^{2} \\
& \leq 4 \pi K_{2}\left|C_{j}\right|^{-1} N^{-1} \sum_{l=1}^{M} N^{-2 d_{l}} \sum_{m \in C_{j}}\left|g_{m}\left(u_{l}\right)\right|^{2} v_{m}^{-2} \\
& =4 \pi K_{2}\left|C_{j}\right|^{-1} N^{-1} \sum_{m \in C_{j}} v_{m}^{-2} \sum_{l=1}^{M} N^{-2 d_{l}}\left|g_{m}\left(u_{l}\right)\right|^{2}=4 \pi K_{2}\left|C_{j}\right|^{-1} N^{-1} \sum_{m \in C_{j}} v_{m}^{-1},
\end{aligned}
$$

so that

$$
\mathbb{E}\left|\widehat{b}_{j k}-b_{j k}\right|^{2} \leq C n^{-1}\left|C_{j}\right|^{-1} \sum_{m \in C_{j}}\left[\tau_{1}(m, n)\right]^{-1}
$$

(One can obtain an upper bound for $\mathbb{E}\left|\widehat{a}_{j_{0} k}-a_{j_{0} k}\right|^{2}$ by following similar arguments.) 
In order to prove (5.7), define

$$
B_{l}=N^{-2 d_{l}}\left[\sum_{l=1}^{M} N^{-2 d_{l}}\left|g_{m}\left(u_{l}\right)\right|^{2}\right]^{-1} .
$$

Note that

$$
\mathbb{E}\left(z_{l m_{1}} z_{l m_{2}} z_{l m_{3}} z_{l m_{4}}\right) \leq\left[\Pi_{i=1}^{4} \mathbb{E}\left|z_{m_{i} l}\right|^{4}\right]^{1 / 4} .
$$

Consequently, using Assumption A1, the fact that $z_{l m}$ are independent for different $l^{\prime}$ s, and that $\mathbb{E}\left|z_{m l}\right|^{4}=3\left[\mathbb{E}\left|z_{m l}\right|^{2}\right]^{2}$ for standard (complex-valued) Gaussian random variables $z_{m l}$, one obtains

$$
\begin{aligned}
\mathbb{E}\left|\widehat{b}_{j k}-b_{j k}\right|^{4} & =O\left(N^{-2} \sum_{l=1}^{M} B_{l}^{4}\left[\sum_{m \in C_{j}}\left|\psi_{m j k}\right|\left|g_{m_{2}}\left(u_{l}\right)\right|\left(\mathbb{E}\left|z_{m l}\right|^{4}\right)^{1 / 4}\right]^{4}\right) \\
& +O\left(\left[N^{-1} \sum_{l=1}^{M} B_{l}^{2} \sum_{m_{1}, m_{2} \in C_{j}} \bar{\psi}_{m_{1} j k} \psi_{m_{2} j k} \overline{g_{m_{1}}\left(u_{l}\right)} g_{m}\left(u_{l}\right) \operatorname{Cov}\left(z_{l m_{1}}, \bar{z}_{l m_{2}}\right)\right]^{2}\right) \\
& =O\left(N^{-2} \sum_{l=1}^{M} B_{l}^{4}\left[\sum_{m \in C_{j}}\left|\psi_{m j k}\right|^{2}\left|g_{m}\left(u_{l}\right)\right|^{2} \sum_{m \in C_{j}} \mathbb{E}\left|z_{m l}\right|^{2}\right]^{2}\right) \\
& +O\left(\left[n^{-1}\left|C_{j}\right|^{-1} \sum_{m \in C_{j}}\left[\tau_{1}(m, n)\right]^{-1}\right]^{2}\right)
\end{aligned}
$$

Since $\sum_{m \in C_{j}} \mathbb{E}\left|z_{m l}\right|^{2}=O\left(\left|C_{j}\right|\right)$, one derives

$$
\begin{aligned}
\mathbb{E}\left|\widehat{b}_{j k}-b_{j k}\right|^{4} & =O\left(\left|C_{j}\right|^{-1} \sum_{m \in C_{j}}\left[\frac{1}{M^{3}} \frac{\tau_{2}(m, n)}{\left[\tau_{1}(m, n)\right]^{4}}\right]+\frac{\Delta_{1}^{2}(j, n)}{n^{2}}\right) \\
& =O\left(M^{-3} \Delta_{2}(j, n)+n^{-2} \Delta_{1}^{2}(j, n)\right) .
\end{aligned}
$$

It is straightforward to show that, when $\alpha_{1}=\alpha_{2}=0$, one has

$$
\Delta_{2}(j, n)=O\left(2^{6 j \nu} j^{3 \lambda_{1}} \varepsilon_{n}^{-3}\right) .
$$

Thus, using (8.1) and the fact that $2^{j} \leq 2^{J-1}<\left(n^{*}\right)^{1 /(2 \nu+1)}$, 8.6) can be rewritten as

$$
\begin{aligned}
\mathbb{E}\left|\widehat{b}_{j k}-b_{j k}\right|^{4} & =O\left(2^{6 \nu j} j^{3 \lambda_{1}} \varepsilon_{n}^{-3} M^{-3}+2^{4 j \nu} j^{2 \lambda_{1}} \varepsilon_{n}^{-2} n^{-2}\right) \\
& =O\left(n^{3}(\ln n)^{3 \lambda_{1}}\left(n^{*}\right)^{-3 /(2 \nu+1)}\right) .
\end{aligned}
$$

Hence, (5.7) follows. This completes the proof of the lemma. 
Proof of Lemma 3, Consider a set of vectors

$$
\Omega_{j r}=\left\{v_{k}, k \in U_{j r}: \sum_{k \in U_{j r}}\left|v_{k}\right|^{2} \leq 1\right\}
$$

and a centered Gaussian process

$$
Z_{j r}=\sum_{k \in U_{j r}} v_{k}\left(\widehat{b}_{j k}-b_{j k}\right)
$$

Note that

$$
\sup _{v} Z_{j r}(v)=\sqrt{\sum_{k \in U_{j r}}\left|\widehat{b}_{j k}-b_{j k}\right|^{2}} .
$$

We shall apply below a lemma of Cirelson, Ibragimov and Sudakov (1976) which states that, for any $x>0$,

$$
\operatorname{Pr}\left(\sum_{k \in U_{j r}}\left|\widehat{b}_{j k}-b_{j k}\right|^{2} \geq\left(x+B_{1}\right)\right) \leq \exp \left(-\frac{x^{2}}{2 B_{2}}\right),
$$

where,

$$
B_{1}=\mathbb{E}\left[\sqrt{\sum_{k \in U_{j r}}\left|\widehat{b}_{j k}-b_{j k}\right|^{2}}\right] \leq \frac{\sqrt{c_{1}} 2^{j \nu} j^{\lambda_{1} / 2} \sqrt{\ln n}}{\sqrt{n^{*}}}
$$

with $c_{1}$ defined in (8.1), and

$$
B_{2}=\sup _{v \in \Omega_{j r}} \operatorname{Var}\left(Z_{j r}(v)\right)=\sup _{v \in \Omega_{j r}} \mathbb{E}\left|\sum_{k \in U_{j r}} v_{k}\left(\widehat{b}_{j k}-b_{j k}\right)\right|^{2} .
$$

Denote

$$
w_{j m}=\sum_{k \in U_{j r}} v_{k} \psi_{m j k}\left[\sum_{l=1}^{M} N^{-2 d_{l}}\left|g_{m}\left(u_{l}\right)\right|^{2}\right]^{-1}, \quad m \in C_{j} .
$$

Then, under Assumption A2 with $\alpha_{1}=\alpha_{2}=0$, using argument similar to the proof of (5.6), one obtains

$$
\begin{aligned}
B_{2} & =\sup _{v \in \Omega_{j r}}\left\{N^{-1} \sum_{m_{1}, m_{2} \in C_{j}} \overline{w_{j m_{1}}} w_{j m_{2}} \mathbb{E}\left[\sum_{l=1}^{M} N^{-4 d_{l}} \overline{g_{m_{1}}\left(u_{l}\right)} g_{m_{2}}\left(u_{l}\right) z_{l m_{1}} \bar{z}_{l m_{2}}\right]\right\} \\
& \leq \sup _{v \in \Omega_{j r}} N^{-1} \sum_{l=1}^{M} N^{-4 d_{l}} \lambda_{\max }\left(\boldsymbol{\Sigma}^{(l)}\right) \sum_{m \in C_{j}}\left|w_{j m} g_{m}\left(u_{l}\right)\right|^{2} \\
& \leq K_{3} n^{-1} \sup _{v \in \Omega_{j r}}\left\{\sum_{m \in C_{j}}\left|w_{j m}\right|^{2}\left[\tau_{1}(m, n)\right]^{-1}\right\} \leq 4 \pi C_{3}^{*} 2^{2 j \nu} j^{\lambda_{1}}\left(n^{*}\right)^{-1},
\end{aligned}
$$


where $C_{3}^{*}=\left(K_{3}\right)^{-1}(\ln 2)^{\lambda_{1}}(2 \pi / 3)^{2 \nu}$.

Apply now inequality (8.7) with $x$ such that $x^{2}=2 B_{2} \kappa \ln n$, and note that

$$
\left(x+B_{1}\right)^{2}=\left(n^{*}\right)^{-1} 2^{2 j \nu} j^{\lambda_{1}} \ln n\left(\sqrt{c_{1}}+\sqrt{8 \pi \kappa K_{3}^{-1}(\ln 2)^{\lambda_{1}}(2 \pi / 3)^{2 \nu}}\right)^{2}
$$

and

$$
\mu^{2} \geq 4\left(1-h_{1}\right)^{-1}\left(\sqrt{c_{1}}+\sqrt{8 \pi \kappa K_{3}^{-1}(\ln 2)^{\lambda_{1}}(2 \pi / 3)^{2 \nu}}\right)^{2},
$$

which guarantees (8.7). This completes the proof of the lemma.

Proof of Theorem 2. Direct calculations yield that under Assumptions A1, A2 and (5.5), for some constants $c_{1}>0$ and $c_{2}>0$, independent of $n$, one has

$$
\Delta_{1}(j, n) \leq \begin{cases}c_{1} \varepsilon_{n}^{-1} 2^{2 \nu j} j^{\lambda_{1}}, & \text { if } \alpha_{1}=\alpha_{2}=0, \\ c_{2} \varepsilon_{n}^{-1} 2^{2 \nu_{1} j} j^{\lambda_{1}} \exp \left\{\alpha_{1}\left(\frac{8 \pi}{3}\right)^{\beta} 2^{j \beta}\right\}, & \text { if } \alpha_{1} \alpha>0 .\end{cases}
$$

Using 8.8, the proof of this theorem is now almost identical to the proof of Theorem 2 in Pensky and Sapatinas (2010). This completes the proof of the theorem.

\subsection{Proofs of the Statement in Section 6 .}

Proof of Lemma 4. Below we consider only the case of $a_{1}>0$. Validity of the satement for $a_{1}=0$ follows from Pensky and Sapatinas (2010).

By direct calculations, one obtains that

$$
\tau_{1}(m, n)=M^{-1}\left(4 \pi^{2} m^{2}\right)^{-1} N^{-2 a_{2}} \sum_{l=1}^{M} q^{2}(l / M) \sin ^{2}\left(2 \pi m l M^{-1}\right) N^{-2 a_{1} l / M} .
$$

Therefore,

$$
\left(4 \pi^{2} m^{2}\right)^{-1} q_{1}^{2} N^{-2 a_{2}} S(m, n) \leq \tau_{1}(m, n) \leq\left(4 \pi^{2} m^{2}\right)^{-1} q_{2}^{2} N^{-2 a_{2}} S(m, n),
$$

where

$$
S(m, n)=M^{-1} \sum_{l=1}^{M} \sin ^{2}\left(2 \pi m l M^{-1}\right) N^{-2 a_{1} l / M} .
$$

Denote $p=N^{-2 a_{1} / M}, x=4 \pi m M^{-1}$ and note that, as $n \rightarrow \infty$,

$$
p^{M}=N^{-2 a_{1}} \rightarrow 0
$$

and

$$
\begin{aligned}
p & =\exp \left(-2 a_{1} M^{-1} \ln N\right) \\
& =1-2 a_{1} M^{-1} \ln N+2 a_{1}^{2} M^{-2} \ln ^{2} N+o\left(M^{-2} \ln ^{2} N\right),
\end{aligned}
$$


since $M^{-1} \ln N \rightarrow 0$ as $n \rightarrow \infty$.

Using the fact that $\sin ^{2}(x / 2)=(1-\cos x) / 2$ and formula 1.353.3 of Gradshtein \& Ryzhik (1980), we obtain

$$
S(m, n)=\frac{1}{M}\left[\frac{1-p^{M}}{1-p}-\frac{1-p \cos x-p^{M} \cos (M x)+p^{M+1} \cos ((M-1) x)}{1-2 p \cos x+p^{2}}\right] .
$$

Since $m$ is an integer and $x=4 \pi m M^{-1}$,

$$
\cos (M x)=1, \quad \sin (M x)=0, \quad \cos ((M-1) x)=\cos x .
$$

Therefore, simple algebraic transformations yield

$$
S(m, n)=\frac{p(p+1)\left(1-p^{M}\right)(1-\cos x)}{M(1-p)\left[(1-p)^{2}+2 p(1-\cos x)\right]}
$$

The asymptotic expansion (8.10) for $p$ as $n \rightarrow \infty$, leads to

$$
\frac{\left(1-p^{M}\right)}{M(1-p)} \approx \frac{1-N^{-2 a_{1}}}{4 a_{1} \ln N\left(1-a_{1} M^{-1} \ln N\right)}
$$

so that, if $N$ is large enough, due to $p<1$, one obtains an upper bound for $S(m, n)$ :

$$
S(m, n)=\frac{\left(1-p^{M}\right)}{M(1-p)}\left[\frac{(1-p)^{2}}{p(p+1)(1-\cos x)}+\frac{2}{p+1}\right]^{-1} \leq \frac{1}{2 a_{1} \ln N} .
$$

In order to obtain a lower bound for $S(m, n)$, we note that for $N$ large enough, one has $1 / 2<p<1$. Consider the following two cases: $x \geq \pi / 3$ and $x<\pi / 3$. If $x \geq \pi / 3$, then $\cos x \leq 1 / 2$ and

$$
F(p, x)=\frac{(1-p)^{2}}{p(p+1)(1-\cos x)}+\frac{2}{p+1} \leq 2
$$

If $x<\pi / 3$, we can use the fact that $1-\cos x=2 \sin ^{2}(x / 2) \geq 3 x^{2} / 8$, so that

$$
F(p, x) \leq \frac{4}{3}\left[1+\frac{8(1-p)^{2}}{3 x^{2}}\right] \leq \frac{4}{3}\left[1+\frac{2 a_{1}^{2} \ln ^{2} N}{3 \pi^{2} m^{2}}\right]
$$

for $N$ large enough.

Since $|m|=C_{m} 2^{j}>C_{m} C_{0} \ln n$ for some $\delta>0$ and $\ln n \geq\left(1-\theta_{1}\right)^{-1} \ln N$ due to assumption (6.1), one has $m^{2} \geq C_{m} C_{0}\left(1-\theta_{1}\right)^{-1} \ln ^{2} N$ and

$$
S(m, n) \geq C(\ln N)^{-1} \text {. }
$$

Observe now that $\ln N \asymp \ln n$. This completes the proof of the theorem. 


\section{Acknowledgements}

Marianna Pensky was supported in part by National Science Foundation (NSF), grant DMS-1106564.

\section{References}

[1] Alvarez-Lacalle, E., Dorow, B., Eckmann, J. P. and Moses, E. (2006). Hierarchical structures induce long-range dynamical correlation in written texts. Proceedings of the National Academy of Sciences, 103, 7956-7961.

[2] Beran, J. (1992). Statistical methods for data with long-range dependence. Statistical Science, 4, 404-416.

[3] Beran, J. (1994). Statistics for Long-Memory Processes. New York: Chapman and Hall.

[4] Beran, J. Feng, Y., Ghosh, S. and Kulik, R. (2013). Long-Memory Processes: Probabilistic Properties and Statistical Methods. New York: Springer-Verlag.

[5] Bunea, F., Tsybakov, A. and Wegkamp, M.H. (2007). Aggregation for Gaussian regression. Annals of Statistics, 35, 1674-1697.

[6] Cheng, B. and Robinson, P. M. (1994). Semiparametric estimation from time series with long-range dependence. Journal of Econometrics, 64, 335-353.

[7] Cirelson, B.S., Ibragimov, I.A. and Sudakov, V.N. (1976). Norm of Gaussian sample function. In Proceedings of the 3rd Japan-U.S.S.R. Symposium on Probability Theory, Lecture Notes in Mathematics, 550 pp. 20-41, Berlin: Springer-Verlag.

[8] Comte, F., Dedecker, J. and Taupin, M. L. (2008). Adaptive density deconvolution with dependent inputs. Mathematical Methods of Statistics , 17, 87-112.

[9] Csörgo, S. and Mielniczuk, J. (2000). The Smoothing dichotomy in random-design regression with long-memory errors based on moving averages. Statistica Sinica, 10, $771-787$.

[10] De Canditiis, D. and Pensky, M. (2004). Discussion on the meeting on "Statistical Approaches to Inverse Problems". Journal of the Royal Statistical Society, Series B, 66, 638-640.

[11] De Canditiis, D. and Pensky, M. (2006). Simultaneous wavelet deconvolution in periodic setting. Scandinavian Journal of Statistics, 33, 293-306.

[12] Doukhan, P., Oppenhein, G. and Taqqu, M.S. (2003). Theory and Applications of Long-Range Dependence. Boston: Birkhaüser. 
[13] Fan, J. and Koo, J. (2002). Wavelet deconvolution. IEEE Transactions on Information Theory, 48, 734-747.

[14] Geweke, J. and Porter-Hudak, S. (1983). The estimation and application of long memory time series models. Journal of Time Series Analysis, 4, 221-238.

[15] Golubev, G. (2004) The principle of penalized empirical risk in severely ill-posed problems. Probability Theory and Related Filelds, 130, 18-38.

[16] Golubev, G.K. and Khasminskii, R.Z. (1999). A statistical approach to some inverse problems for partial differential equations. Problems of Information Transmission, 35, 136-149.

[17] Grenander, U. and Szegö, G. (1958). Toeplitz Forms and their Applications. California Monographs in Mathematical Sciences, Berkeley-Los Angeles: University of California Press.

[18] Gradshtein, I.S. and Ryzhik, I.M. (1980). Tables of Integrals, Series, and Products. New York: Academic Press.

[19] Harsdörf, S. and Reuter, R. (2000). Stable deconvolution of noisy lidar signals. In Proceedings of EARSeL-SIG-Workshop LIDAR, Dresden/FRG, June 16-17.

[20] Karagiannis, T., Molle, M. and Faloutsos, M. (2004). Long-range dependence, ten years of internet traffic modeling. IEEE Internet Computing, 8, 57-64.

[21] Karmeshu, D. and Krishnamashari, A. (2004). Sequence variability and long-range dependence in DNA. IEEE Internet Computing, 8, 1354-1361.

[22] Kulik, R. (2008). Nonparametric deconvolution problem for dependent sequences. Electronic Journal of Statistics, 2, 722-740.

[23] Kulik, R. and Raimondo, M. (2009). $L^{p}$ wavelet regression with correlated errors. Statistica Sinica, 19, 1479-1489.

[24] Lattes, R. and Lions, J.L. (1967). Methode de Quasi-Reversibilite et Applications. Travoux et Recherche Mathematiques, 15. Paris: Dunod.

[25] Lo, A. (2001). Fat tails, long memory and the stock market since the 1960s. Economic Notes, Banca Monte dei Paschi di Siena.

[26] Mielniczuk, J. and Wu, W. B. (2004) On random-design model with dependent errors. Statistica Sinica, 14, 1105-1126 .

[27] Navarro, F., Chesneau, C., Fadili, J., and Sassi, T. (2013) Block thresholding for wavelet-based estimation of function derivatives from a heteroscedastic multichannel convolution model. Electron. J. Statist., 7, 428-453. 
[28] Painter, S. (1998). Long-range dependence in subsurfaces: empirical evidence and simulation methods. Invited paper at the American Geophysical Union, Fall Meeting, 1998.

[29] Park, Y.J., Dho, S.W. and Kong, H.J. (1997). Deconvolution of long-pulse lidar signals with matrix formulation. Applied Optics, 36, 5158-5161.

[30] Pensky, M. and Sapatinas, T. (2009). Functional deconvolution in a periodic setting: uniform case. Annals of Statistics, 37, 73-104.

[31] Pensky, M., Sapatinas, T. (2010). On convergence rates equivalency and sampling strategies in a functional deconvolution model. Annals of Statistics, 38, 1793-1844.

[32] Pensky, M., Sapatinas, T. (2011) Multichannel boxcar deconvolution with growing number of channels. Electronic Journal of Statistics, 5, 53-82.

[33] Pensky, M. and Vidakovic, B. (1999). Adaptive wavelet estimator for nonparametric density deconvolution. Annals of Statistics, 27, 2033-2053.

[34] Tsybakov, A.B. (2008). Introduction to Nonparametric Estimation, New York: Springer-Verlag.

[35] Van Zanten, H. and Zareba, P. (2008). A note on wavelet density deconvolution for weakly dependent data. Statistical Inference for Stochastic Processes, 11, 207-219.

[36] Varotsos, C. and Kirk-Davidoff, D. (2006). Long-memory processes in global ozone and temperature variations at the region 60S-60N. Atmospheric Chemistry and Physics, 6, 4093-4100.

[37] Walter, G. and Shen, X. (1999). Deconvolution using Meyer wavelets. Journal of Integral Equations and Applications, 11, 515-534.

[38] Wang, Y. (2012). Minimax estimation via wavelets for indirect long-memory data. Journal of Statistical Planning and Inference, 64, 45-55.

[39] Wishart, J. M. (2012). Wavelet deconvolution in a periodic setting with long-range dependent errors. Journal of Statistical Planning and Inference, 143, 867-881.

[40] Yang, Y. (2001). Nonparametric regression with dependent errors. Bernoulli, 7, 633655. 